Journal of Ancient Philosophy Vol. II 2008 Issue 2

\title{
Ressemblance et Participation chez Platon
}

Barbara Botter (PUC-RJ)

In this text we propose to clarify the problem of participation in Plato, namely the causal relationship between the Ideas and the sensible objects. If the existence of a causal relation between image and model is easy to find, the nature of the relationship is aporetic, a source of perplexity. This statement is a reason to suppose that the dilemma of participation and the criticism of Ideas in the Parmenides that depend on it, are put neither as trivial fallacies nor fatal objections, but as problems to be solved.

The solution that we suggest is that the separation between Ideas and the sensible objects is not a symmetrical relation: sensibles are wholly dependant images of Ideas, they are neither individual nor substantial. The separation is not a symmetrical relation, because the Ideas are separate from sensibles in that they are capable of existing apart from sensibles; sensibles are not separate from Ideas in that they are incapable of existing apart from Ideas.

\section{Introduction}

Dans ce texte nous nous proposons d'élucider le problème de la participation chez Platon, à savoir la relation causale entre les Formes et les sensibles. Platon définira la Forme comme la cause des sensibles dans le Phédon ${ }^{1}$ ou dans 1'Hippias Majeur ${ }^{2}$. Si l'existence d'une relation causale entre les Formes et les sensibles ne fait pas de doute, par contre la nature de cette relation demeure énigmatique. La question, pourtant, sera comment comprendre ce lien causal. Ce problème constitue le point de départ de notre réflexion.

Dans le Timée, le Banquet, l'Euthydème et la République on retrouve des variations terminologiques en relation à l'indication de ce lien causal; en revanche, le Parménide semble aborder le rapport d'une forme différente. Ces variations nous semblent refléter le caractère extrêmement problematique du rapport Formes - sensibles.

\footnotetext{
${ }^{1}$ Platon, Phédon 100d.

${ }^{2}$ Platon, Hippias Majeur 287c.
} 


\section{Journal of Ancient Philosophy Vol. II 2008 Issue 2}

À partir de l'étude de la Ière partie du Parménide nous nous proposons de comprendre les Formes comme des Modèles dont les sensibles seraient les images. Le rapport de participation se comprend alors comme un rapport de ressemblance, d'imitation, mais d'une forme non commune, c'est à dire d'une forme dissymétrique. La dissymétrie du rapport sensible - Forme est dûe à la différence de nature entre les composants : l'un dépendent d'autre chose que lui-même, l'autre existent en lui-même et par lui-même. Cette dissymétrie explique l'unilatéralité de la relation: seuls les sensibles participent des Formes.

\section{$\S$ 1) La Forme comme cause des sensibles}

La Forme platonicienne semble partager avec le concept communément entendu quelque point commun. Le concept désigne une idée abstraite et générale, déduite du sensible en tant qu'elle est résultat d'une abstraction; son élaboration comme la saisie des Formes par l'esprit humain débutent par une confrontation à la diversité sensible. Mais si le concept est construit par l'homme, les Formes en revanche s'imposent comme des réalités transcendantes et autonomes au terme de l'ascension dialectique. Aussi, si le beau était un concept, on pourrait dire que c'est parce qu'il existe de belles choses que le beau existe, mais Platon définira plutôt le Beau d'une manière différente, à savoir comme la cause de la beauté des choses : «C'est par le Beau que toutes les choses belles sont belles $»^{3}$.

Le datif $\tau \hat{\widehat{\omega}} \kappa \alpha \lambda \hat{\omega}$ (c'est par le Beau) est sans doute un datif causal. Or, il y a deux problèmes : 1) le problème de savoir quel sens se cache sous ce datif causal «c'est par le Beau...»; 2) le problème de la nature de la relation entre la Forme «Beau » et les choses belles.

En ce qui concerne 1), nous ne pouvons pas nous occuper maintenant d'une façon approfondie. En tout cas, il faut mieux expliquer où se cache la difficulté. Comme le dit justement Natali : «Il problema di questa narrazione è se Socrate, quando parla di un ê̂dos tês aitías [ovvero di una specie di causa] che lui si sarebbe fabbricato ... intende parlare di un genere di causa diversa da quella che finora abbiamo visto, o se quella accezione di aitia che pervade tutto il corpus platonico, cioè, l'idea di "un ente che, per

\footnotetext{
${ }^{3}$ Platon, Phédon 100d. On retrouve la même formule dans l'Hippias Majeur 287c avec l'exemple de la justice.
} 
mezzo del suo operare, produce un effetto", continua ad essere usata anche qui $\gg{ }^{4}$. Les interprètes ne sont pas d'accord sur ce point. Vlastos, par exemple, pense que ici Platon se réfère à une « cause formale », à savoir l'idée représente l'essence de la chose $\mathrm{e}^{5}$. Il y a d'autres interprètes qui attribuent à l'idée platonicienne une fonction au même temps motrice, finale, et formale ${ }^{6}$. Il y en a d'autres qui interprètent ce rapport comme une relation de causalité efficiente: «le idee trasmettono effettivamente qualcosa che determina una differenza essenziale nelle cose empiriche $»^{7}$.

Le second problème est ce que nous intéresse plus particulièrement. Dans le cas du Phédon, on peut penser que l'expression se réfère à une formule précédente selon laquelle une chose est belle puisque elle participe $(\mu \varepsilon \tau \varepsilon \dot{\chi \varepsilon})$ du Beau ${ }^{8}$. En ce cas comment comprendre la «participation »? Socrate même est très hésitant quand il essaie d'expliquer cette appellation: il s'agit de savoir si cette relation est une

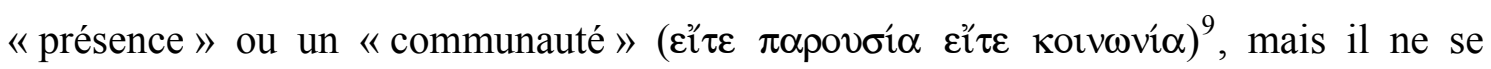
prononcera pas : «sur ce dernier point en effet je ne prends point jusqu'à présent fermement parti, mais bien sur celui-ci que le Beau est ce qui rend belles toutes les belles choses $\gg{ }^{10}$. Sans doute il y a une relation entre les Formes et les sensibles, mais que le Beau soit cause parce qu'il est « présent », à savoir immanent, ou parce qu'il y a « communauté », à savoir réciprocité entre Formes et sensibles, est bien difficile à savoir.

Dans plusieurs dialogues ${ }^{11}$ il y a des variations terminologiques entre les termes

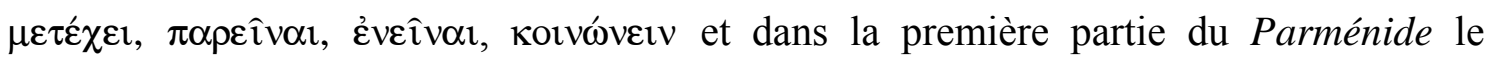
philosophe semble employer indifféremment $\mu \varepsilon \tau \varepsilon \dot{\varepsilon} \chi \imath v$ et $\mu \varepsilon \tau \alpha \lambda \alpha \mu \beta \alpha ́ v \varepsilon \imath v$ pour désigner la même relation de participation. Une autre chose, à notre avis, surprenant est que dans le Timée Platon aborde la relation que nous intéresse en expliquant l'ordre du monde, mais il n'emploie pas les termes cités. Platon affirme que les sensibles imitent les Formes, ils en sont les images : «les choses qui entrent en lui ( $\chi \omega \dot{\rho} \alpha)$ et qui en sortent

\footnotetext{
${ }^{4}$ Natali 2003, p. 167.

${ }^{5}$ Cfr. Vlastos 1981.

${ }^{6}$ Zeller 1922, p. 687.

${ }^{7}$ Fronterotta 2000, p. 218 ; cfr. Natali 2003 ; Sedley 1998.

${ }^{8}$ Platon, Phédon 100c; cfr. G. Vlastos 1973, p. 87, n.33.

${ }^{9}$ Platon, Phédon 100c-101c.

${ }^{10}$ Platon, Phédon 100d.

${ }^{11} \mathrm{Vd}$. Banquet, 211b; Euthydème, 301a; République, III 402c et 476a.
} 
sont des imitations ( $\mu \mu \eta \dot{\eta} \mu \alpha \tau)$ de réalités éternelles ${ }^{12}$. Malheureusement Platon ne précise pas dans le Timée la nature de cette imitation, cependant nous avons à notre disposition un dialogue susceptible de nous guider dans cette recherche, puisque il pose explicitement le problème de la participation : la première partie du Parménide ${ }^{13}$.

Ce texte est essentiel pour deux raisons : 1) parce qu'il met à l'épreuve l'existence des Formes ; 2) pour sa ressemblance frappante avec l'explication de l'ordre du monde du Timée : il propose de comprendre les Formes comme des modèles dont les sensibles sont images; le rapport de participation se comprenant alors comme un rapport de ressemblance ${ }^{14}$.

Cette hypothèse permet-elle de progresser dans la compréhension du rapport de participation ou la participation est réductible à une métaphore vide de sens, comme le

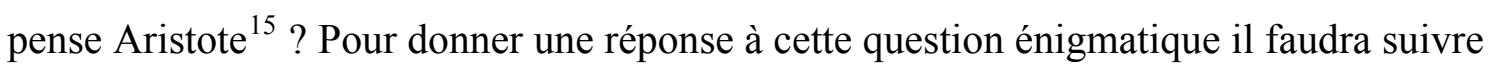
l'enseignement du jeune Socrate par Parménide au cours duquel Socrate émet l'hypothèse de la participation comme rapport de ressemblance bien que son maître la réduise à une aporie.

\section{$\S$ 2) Les apories de la participation}

Dans la première partie du Parménide la théorie de l'existence des Formes et la relation de telles Formes avec les sensibles particuliers soutenue par Socrate est mise à l'épreuve par Parménide. Les critiques les plus redoutables portent sur le problème de la

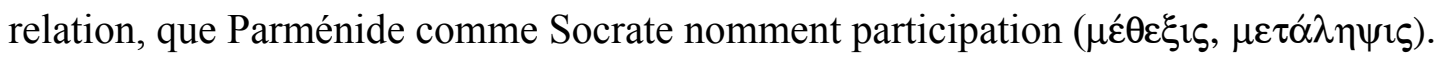

Ier argument : le dilemme de la participation

La première critique que Parménide présente est ce que Brisson nomme « dilemme de la participation ${ }^{16}$ : les choses participent-elles à la totalité de la Forme ou seulement à une partie d'elle ${ }^{17}$ Socrate ne peut pas soutenir la première partie du

\footnotetext{
${ }^{12}$ Platon, Timée, 50c.

${ }^{13}$ Platon, Parménide, Ière partie, jusqu'à $135 \mathrm{~d}$.

${ }^{14}$ Platon, Timée, 132d1-8.

${ }^{15}$ Aristote, Metaph., A, 9 991a20-22.

${ }^{16}$ L'expression est employée par L. Brisson et par R. E. Allen (Brisson 1994, p. 36; Allen 1983 p. 113).

${ }^{17}$ Platon, Parm., 131 a-c.
} 
Journal of Ancient Philosophy Vol. II 2008 Issue 2

dilemme sans mettre en péril l'unité de la Forme, puisque si la Forme est en totalité en chacune des choses qui participent d'elle, alors la Forme est distincte d'elle-même. Socrate tente une solution différente : peut-être la Forme est comme le jour qui « restant un et identique, se trouve en plusieurs endroits en même temps et n'en est pour si peu distinct de lui-même ${ }^{18}$. Parménide répond a cela en utilisant une comparaison différente, qui mène au problème de la divisibilité de la Forme ${ }^{19}$ : un voile reste un lorsqu'il est étendu sur plusieurs choses, mais chacune des choses qui est sous ce voile est couverte par une partie du voile. Cela signifie que les choses qui participent d'une Forme ont chacune une part de cette Forme, ce qui implique que la Forme soit divisible. Socrate, loin d'accepter la divisibilité des Formes, met en évidence les conséquences absurdes d'une telle possibilité, par exemple dans le cas de la Grandeur : si certaines choses grandes sont grandes puisque elles participent $\mathrm{de}^{20}$ la Grandeur, à savoir à une partie de cette Forme, alors la partie étant plus petite que le tout, ces choses seront grandes en vertu d'une partie de la Grandeur plus petite que la Grandeur.

Le caractère sophistique de cet argument ne permet pas de sortir de cet aporie et Socrate laisse la participation dans l'obscurité.

\section{IIème argument : l'Argument du Troisième Homme}

L'argument suivant est connu sous le nom d' "Argument du Troisième Homme » ${ }^{21}$ et mène au regressus infini ${ }^{22}$ : quand plusieurs objets nous apparaissent comme étant grands, nous voyons dans l'ensemble de ces objets un caractère identique, la Grandeur. Or, pour que l'on puisse subsumer ces objets grands sous le caractère unique Grandeur $(\mathrm{G})$, il faut que celui-ci ait la même caractéristique que les objets, à savoir que la Grandeur soit grande. On se retrouve donc en présence de deux groupes : la Grandeur G et les objets grands. Ainsi, il faut reconnaître que les deux groupes sont grands grâce à un nouveau caractère commun, la Grandeur $\mathrm{G}^{2}$. Or, puisque on peut poursuivre le raisonnement à l'infini, la Forme Grandeur se multipliera indéfiniment. Donc Parménide peut conclure : " il y aura encore une Forme, différente, en vertu de laquelle

\footnotetext{
${ }^{18}$ Platon, Parm., 131b. Nous citons la traduction de L. Brisson.

${ }^{19}$ Platon, Parm., $131 \mathrm{c}-\mathrm{e}$.

${ }^{20}$ Nous disons “de" la Grandeur parce que Parménide emploie dans ce passage un génitif partitif plutôt que l'accusatif.

${ }^{21}$ Cfr. Aristote, Metaph., A 9, 990 b15 et M 4, 1079a11.

${ }^{22}$ Platon, Parm., 132a-b.
} 
Journal of Ancient Philosophy Vol. II 2008 Issue 2

la Grandeur en soi et les choses que en participent seront grandes. Par suite, chacune de tes Formes ne sera désormais plus une, mais elle se multipliera sans limite ${ }^{23}$.

Socrate est résolu à défendre la participation, mais il est en même temps incapable de mettre en cause les arguments de son maître. Il propose alors deux nouvelles hypothèses. Nous n'étudierons pas la première, selon laquelle les Formes sont des pensées $(v o \eta ́ \mu \alpha \tau \alpha)$ qui se trouvent dans les âmes ${ }^{24}$, nous étudierons précisément la seconde hypothèse ${ }^{25}$, qui est le dernière tentative de Socrate pour défendre son idée: il propose de concevoir la participation comme un rapport de ressemblance entres les Formes (modèles) et les sensibles (images). Mais on verra que cette hypothèse, reprise par Parménide, mène à un échec, puisque elle introduit un regressus infini.

Ainsi, aucune des tentatives d'explication de la participation par Socrate n'aboutit à la moindre solution. Mauvais augure pour notre étude. A défaut de pouvoir résoudre le problème de la participation, on peut se demander quelle était l'intention de Platon mettant en scène un tel échec.

Le problème est le suivant: les critiques de Parménide doivent être considérées comme des réfutations fatales de la théorie des Formes ou, comme le dit Brisson, «l'inventaire des apories doit être surmonté par celui qui comme Socrate veut maintenir la doctrine des Formes $»^{26}$ ? Cette dernière interprétation est proche de celle de Proclus qui voit dans le Parménide une exhortation à l'exercice dialectique nécessaire pour être capable de soutenir l'existence de telles Formes. Aussi « le procédé $d u$ dialogue est maïeutique et non réfutatif ${ }^{27}$. Cette interprétation nous semble convaincante. Comme le fait remarquer L. Brisson ${ }^{28}$, Parménide ne qualifie jamais ses propres critiques de « réfutations », mais toujours d' «apories ${ }^{29}$. La preuve est, à notre avis, la reprise du dilemme que nous intéresse dans le Philèbe ${ }^{30}$, où le problème $\mathrm{y}$ est qualifié d'aporie, mais Socrate a conscience que cette aporie serait levée si le problème était posé en d'autres termes. Le commentaire éclairant de E. Allen est le suivant: « The Philebus suggests that the dilemma is aporetic, a source of perplextity if

\footnotetext{
${ }^{23}$ Platon, Parm., 132b.

${ }^{24}$ Platon, Parm., 132b-c.

${ }^{25}$ Platon, Parm., 132c-133a.

${ }^{26}$ Brisson 1994, p. 43.

${ }^{27}$ Proclus, Commentaire sur le Parménide, $\S 63$, p. 310 de la trad. Chaignet (vol. I).

${ }^{28}$ Brisson 1994, p. 31

${ }^{29}$ Platon, Parm., 129e5; 130c3; $135 \mathrm{a} 3$.

${ }^{30}$ Platon, Philèbe, $15 \mathrm{~b}-\mathrm{c}$.
} 
Journal of Ancient Philosophy Vol. II 2008 Issue 2

the wrong admissions are made, and of easy passage, given the rights ones. This statement is a reason to suppose that the Dilemma of Participation, and the criticism of Ideas in the Parmenides that depend on it, are put neither as trivial fallacies nor fatal objections, but as problems to be solved ${ }^{31}$. En effet, la deuxième partie du dialogue est constituée par un exercice dialectique : "exerce-toi, pendant que tu es jeune encore! [...] Sinon, la vérité se dérobera à tes proses $»^{32}$. Les Formes sont aussi impensables que nécessaires par cette même personne que les critiques. En effet, explique Parménide, s'il n'y a pas une Forme éternelle correspondant aux choses périssables, c'est la raison qui perde son objet: «si, Socrate, il se trouve quelqu'un qui, au vue de toutes les difficultés qui viennent d'être soulevées [...] n'admette point qu'il y ait des Formes des choses, [...] cet individu ne saura de quel côté tourner sa pensée, parce qu'il n'admet point que pour chaque chose il y a une Forme qui est toujours la même ; et, par suite, il détruira toute possibilité de pratiquer la dialectique ${ }^{33}$. C'est donc bien Parménide qui pose la nécessité des Formes, mais l'inextricable aporie de leur rapport au sensible lui fait conclure désastreusement qu'il y a une totale séparation ( $\chi \omega p ı$ rós) entre Formes et choses ${ }^{34}$. Or, cette hypothèse implique la négation de toute relation, donc l'existence de deux ordres de réalité radicalement distincts. Ces Formes sont des réalités « en soi », soutient Socrate, ce qui signifie qu'aucune d'elles ne se trouve « chez nous », explique Parménide ${ }^{35}$. Aussi, les Formes sont ce qu'elles sont en vertu de leurs relations mutuelles, et les choses se définissent par les relations qu'elles entretient les unes avec les autres ${ }^{36}$. Si donc «les choses sensibles n'ont pas plus d'efficace sur les Formes que les Formes n'en ont sur les choses sensibles ${ }^{37}$, il s'ensuit que la Science en soi n'est science que des Formes, et la science de notre monde n'est science que du «genre d'êtres qui se rencontre dans notre monde ${ }^{38}$. Par conséquence les Formes seront inconnaissables pour nous et la science en soi, étant la plus parfaite, sera la

\footnotetext{
${ }^{31}$ Allen 1983, p. 113.

${ }^{32}$ Platon, Parm., 135d.

${ }^{33}$ Platon, Parm., 135b-c; cfr. Cratyle, 439b-440c.

${ }^{34}$ Platon, Parm., 133a-134e.

${ }^{35}$ Platon, Parm., 133c.

${ }^{36}$ Platon, Parm., 133d-e.

${ }^{37}$ Platon, Parm., 133e. Plus loin Parménide dira: « les unes et les autres n’ont d'efficace que sur les choses qui se trouvent à leur niveau respectif » (134d).

${ }^{38}$ Platon, Parm., 134b.
} 
Journal of Ancient Philosophy Vol. II 2008 Issue 2

science d'une divinité et n'aura pas accès aux choses sensibles ${ }^{39}$. Voilà une conséquence bien difficile à admettre : la privation à une divinité de toute maîtrise sur le monde et l'incapacité de l'homme de connaître les Formes. Si cette conséquence n'est pas inconcevable, elle ruine l'intérêt même de l'hypothèse des Formes, donc l'existence des Formes doit être maintenue, aussi comme le rapport avec le sensible : « Ce serait un homme particulièrement doué, celui à qui on pourrait faire comprendre qu'il y a de

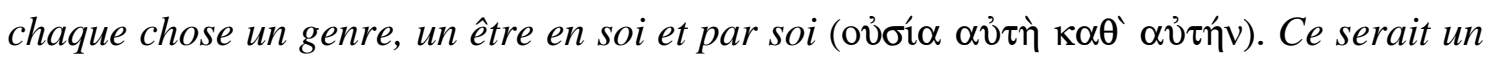
homme merveilleux encore celui qui aurait fait cette découverte et qui pourrait la transmettre par l'enseignement à quelqu'un d'autre après en avoir auparavant éprouvé tous les détails par une critique adéquate ${ }^{40}$. L. Brisson voit dans cette phrase une preuve que Platon ne considérait pas les critiques de Parménide comme fatales. Si en effet il peut concevoir un homme si talentueux qu'il pourrait montrer que les critiques invoquées contre les Formes ne sont pas valides, c'est qu'il considère que ces critiques sont surmontables et que on doit maintenir l'existence des Formes. Mais il y a encore une pointe de scepticisme: Platon pensait-il vraiment que cet homme si merveilleux puisse démontrer l'existence des Formes ou, loin de pouvoir être démontrées, elles ne peuvent être que postulées ? «Poser les Formes c'est, si l'on peut dire, faire un acte de foi scientifique. Les objets sensibles provoquent, comme cause occasionnelle, la réminiscence, mais les Formes ne sont pas «extraites » des sensibles; leur position est exigée par elles-mêmes », écrit Goldschmidt ${ }^{41}$.

Enfin, de la première partie du Parménide se dégagent deux idées essentielles : 1) l'hypothèse des Formes s'impose comme une nécessité ; 2) la séparation totale entre Formes et objets sensibles est insoutenable. La conclusion est la suivante: la participation est une nécessité. La question de la nature de cette relation de participation en est ainsi d'autant plus pressante. Il nous reste à étudier la dernière hypothèse du jeune Socrate : la participation comme imitation.

\section{§ 3) L'hypothèse du jeune Socrate}

\footnotetext{
${ }^{39}$ Cfr. Platon, Parm., 134e.

${ }^{40}$ Platon, Parm., 135a-b.

${ }^{41}$ Goldschmidt 1949, p. 36.
} 
Journal of Ancient Philosophy Vol. II 2008 Issue 2

L'hypothèse du jeune Socrate est formulée ainsi : «Voici, Parménide, ce qui me semble être, à moi, la meilleure explication. Alors que ces Formes sont comme des modèles ( $\left.\pi \alpha \rho \alpha \delta \varepsilon^{\prime} \gamma \mu \alpha \tau \alpha\right)$ qui subsistent dans leur nature, les autres choses entretiennent

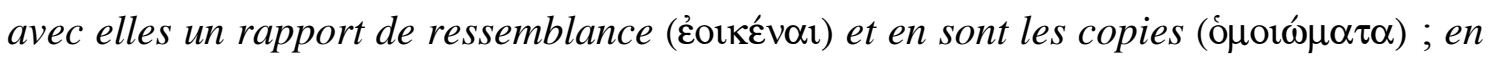
outre, la participation que les autres choses entretiennent avec les Formes n'a pas

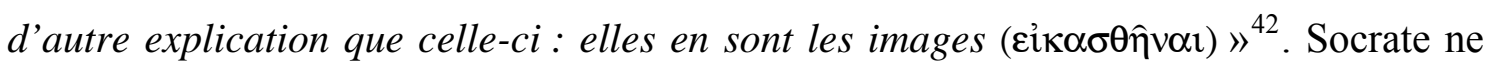

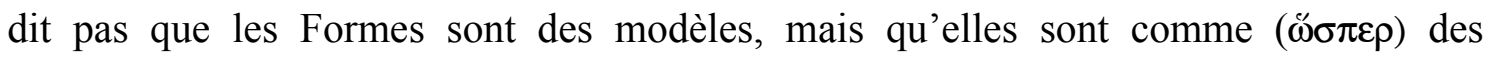
modèles. La comparaison est la suivante $:$ sensibles/Formes $=$ images/Modèles.

Parménide développe l'hypothèse de Socrate et la mène à l'aporie, en y intégrant deux nouvelles idées $^{43}$ : 1) la symétrie du rapport de ressemblance; si l'image ressemble à son modèle, alors le modèle ressemble à son image : «Si quelque chose ressemble à une Forme, cette Forme peut-elle ne pas être semblable à ce qui lui ressemble [...] ? ou est-il quelque moyen par lequel le semblable puisse ne pas être semblable au semblable» - «Il n’y en a point», répond Socrate; 2) une certaine définition de la ressemblance selon laquelle deux choses se ressemblent si elles participent toutes deux de la même Forme : «N'est-il pas absolument nécessaire que ce qui est semblable participe à une seule et même Forme ? - Certainement », répond Socrate. Les deux assertions intégrées à l'hypothèse de Socrate entraînent un regressus infini : «au-delà de cette Forme une autre Forme toujours surgira. Et si cette dernière se trouve ressembler à quelque chose, une autre Forme surgira encore, et jamais ne cessera l'apparition d'une Forme chaque fois nouvelle ${ }^{44}$. Face à cette reformulation de l'Argument du Troisième Homme ${ }^{45}$, Socrate apparemment accepte l'échec : «Ce n'est donc point en vertu de la ressemblance que les autres choses participent aux

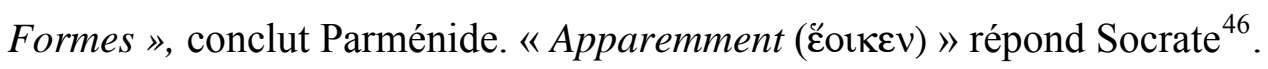

Or, il nous semble que ce sont les deux assertions de Parménide qui entraînent le regressus. Le problème est de savoir dans quelle mesure elles sont légitimes : étaientelles contenues dans l'hypothèse de Socrate ou bien elles sont ajoutées par Parménide? Avant de répondre à cette question, nous devons nous occuper de l'hypothèse de Socrate. Selon Proclus, étant le procédé du dialogue maïeutique, il faut considérer cette

\footnotetext{
${ }^{42}$ Platon, Parm., 132d.

${ }^{43}$ Platon, Parm., 132d-133a.

${ }^{44}$ Platon, Parm., 132e-133a.

${ }^{45}$ Cfr. Platon, Parm., 132a-b.

${ }^{46}$ Platon, Parm., 133a.
} 
hypothèse comme un progrès de l'argumentation de Socrate : «il est évident que la méthode de ces entretiens est la méthode maïeutique, et non un système de critique et de réfutations ; car il n'aurait pas représenté l'interlocuteur faisant des progrès dans la recherche et arrivant à des concepts plus parfaits [...]. L'hypothèse de Socrate est un progrès vers la vérité » ${ }^{47}$.

A notre avis, si cette hypothèse est pertinente, elle doit être en accord avec d'autres dialogues. Nombreux sont les textes qui présentent les Formes comme des Modèles et les sensibles comme images ou imitations. Le textes antérieurs au Parménide nous montrent que Platon n'a pas mis innocemment cette hypothèse dans la bouche de Socrate ; les textes postérieurs confirment la lecture selon laquelle Platon ne considère pas les critiques comme des réfutations. L. Brisson confirme : «A moins de considérer Platon comme un penseur particulièrement pervers ou de supposer un autre ordre de composition des dialogues, il est difficile de penser qu'il a après le Parménide abandonné l'hypothèse de l'existence de Formes transcendantes assimilées à des paradigmes $»{ }^{48}$.

Les textes faisant écho à l'hypothèse du jeune Socrate sont les suivants ${ }^{49}$ :

- dans l'Euthyphron Socrate, ayant admis qu'il existe « un caractère unique par lequel les choses impies sont impies et les choses pieuses sont pieuses », demande à Euthyphron de lui enseigner quel est ce caractère, afin que, dit-il, « je tienne mes yeux fixés dessus et m'en serve comme de modèle ( $\pi \alpha \rho \alpha ́ \delta \varepsilon \imath \gamma \mu \alpha)$, et que si, parmi tes actes ou ceux d'autrui, il en est qui soient conformes à ce modèle, je les déclare pieux, et, s'ils ne le sont pas, impies » ${ }^{50}$.

- Dans le Cratyle la Forme de la navette est le modèle sur lequel le menuisier fabricant une navette fixera son regard ${ }^{51}$.

- Dans le Phédon les Formes sont dites être l'objet d'une réminiscence facilitée par la vue des sensibles qui leur ressemblent $(\pi \rho 0 \sigma \varepsilon o \iota \kappa \varepsilon ́ v \alpha \imath)^{52}$. Si nous nous

\footnotetext{
${ }^{47}$ Proclus, Comm. sur le Parm., § 78, pp. 63-64 de la trad. Chaignet (vol. II).

${ }^{48}$ Brisson 1994, pp. 30-31.

${ }^{49}$ Nous adoptons la chronologie des œuvres due aux travaux de L. Brandwood 1990.

${ }^{50}$ Platon, Euthyphron, 6e.

${ }^{51}$ Platon, Cratyle, 389a-b.

${ }^{52}$ Platon, Phédon, 74e. Socrate emploie le même verbe dans son hypothèse du Parménide 132d.
} 
Journal of Ancient Philosophy Vol. II 2008 Issue 2

souvenons de l'Egalité en soi en voyant deux bouts de bois égaux, c'est qu'il y a un rapport de ressemblance entre l'Egalité en soi e l'égalité sensible ${ }^{53}$.

- $\quad$ L'analogie qui nous intéresse est très présente dans la République ${ }^{54}$.

- $\quad$ On retrouve dans le Phèdre les mêmes considérations que dans le Phédon ${ }^{55}$.

- Enfin le Timée est pour nous d'une importance capitale, puisque la cosmogonie qui y est présentée repose sur cette hypothèse. Le monde a été ordonné par un Démiurge qui a agi en fixant son regard sur un Modèle ${ }^{56}$. Or, connaître le Modèle sur lequel le monde a été conçu, c'est connaître la cause ( $\left.\alpha i \tau^{\prime} \alpha\right)$ du monde. Est-ce le Modèle engendré et corruptible ou le Modèle éternel ? La réponse n'admet pas de doute: le monde étant «la plus belle des choses engendrées » et le Démiurge « la meilleure des causes » ${ }^{57}$, il est évident que le Démiurge a agi conformément au Modèle éternel. Cette conception implique que ce monde-ci soit image de quelque chose d'autre : «il est nécessaire que

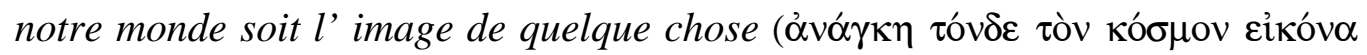
$\varepsilon v \alpha \mathrm{l}) »^{58}$.

Il y a une autre partie du Timée que ira nous intéresser plus loin, à savoir les pages sur la chora ${ }^{59}$, puisque « les choses qui entrent en lui (chora) et qui en sortent sont des

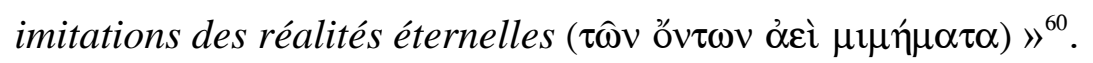

Toutes ces références nous invitent à prendre en considération sérieusement l'hypothèse du jeune Socrate. Dans son étude entièrement consacré à cette analogie $\mathrm{R}$. A. Patterson écrit : « the dialogues in which the theory is most explicit ${ }^{61}$ leave no doubt that Plato held an image-model theory of participation ${ }^{62}$. Mais les témoignages de cette conception ne s'arrêtent pas à l'œuvre de Platon. Aristote atteste une telle conception de la participation dans la Métaphysique: «Quant à cette participation, Platon ne modifiait que le nom : les Pythagoriciens en effet, disent que les êtres existent

\footnotetext{
${ }^{53}$ Platon, Phédon, 74b-75d.

${ }^{54}$ Platon, République, 476c-d; 509e-511e; 514a-516e; 520c; 596b-598d.

${ }^{55}$ Platon, Phèdre, 250a-b; 251a.

${ }^{56}$ Platon, Timée, $27 \mathrm{~d}-29 \mathrm{c}$.

${ }^{57}$ Platon, Timée, 29a.

${ }^{58}$ Platon, Timée, 29b. D’autres formulations associent explicitement les Formes aux Modèles et les sensibles aux images $(37 \mathrm{c} ; 39 \mathrm{e} ; 48 \mathrm{e}-49 \mathrm{a})$

${ }^{59}$ Platon, Timée, $48 \mathrm{e}-52 \mathrm{~d}$.

${ }^{60}$ Platon, Timée, $50 \mathrm{c}$.

${ }^{61}$ Il s'agit du Timée, de la République et du Phédon.

${ }^{62}$ Patterson 1985, pp. 27-28.
} 
par imitation des nombres; pour Platon c'est par une participation, le mot seul est changé ${ }^{63}$. A ce point l'hypothèse du jeune Socrate nous apparaît comme étant vraiment digne d'intérêt. Maintenant nous pouvons exploiter la richesse qu'offre la structure analogique image/Modèle - sensible/Forme.

\section{§ 4) Le statut de la ressemblance}

Dans le Cratyle Socrate propose une définition de l'image mettant en évidence le « principe de non identité ${ }^{64}$. A Cratyle qui conçoit la «bonne image » comme étant celle qui imite le plus de caractères possibles du modèle, Socrate répond : « je crains que la justesse ne soit autre chose et qu'au contraire il ne faille pas du tout reproduire tous les traits de l'objet imité, si l'on veut obtenir une image. Vois si ce que je dis est juste. Y aurait-il deux objets, tels que Cratyle et l'image de Cratyle, si quelque divinité, non contente d'imiter ta couleur et ta forme, comme les peintres, reproduisait aussi tout l'intérieur de ta personne, tel qu'il est, lui donnant la même mollesse et la même chaleur, et y mettait le mouvement, l'âme et la pensée, tels qu'ils sont en toi, en un mot plaçait à côté de toi un double de toutes tes qualités ? Y aurait-il, en ce cas, Cratyle et une image de Cratyle, ou deux Cratyle ? - il me semble à moi, Socrate, qu'il y aurait deux Cratyle - tu vois donc, mon ami, qu'il faut chercher un autre genre de justesse pour l'image et pour les noms, [...], et ne pas vouloir à toute force que l'image cesse d'en être une, si l'on en ôte ou si l'on y ajoute quelque détail. Ne sens-tu pas de

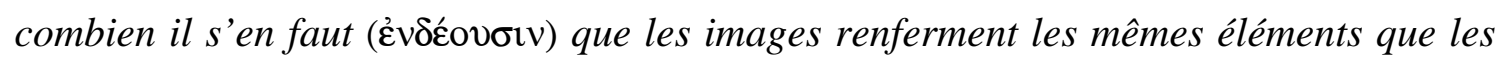
originaux qu'elles imitent ? ${ }^{65}$.

Il est claire que l'image d'une chose n'est pas une reproduction du même, aussi comme l'image de Cratyle n'est pas une autre Cratyle, mais autre chose que Cratyle. Si une chose et son image étaient les mêmes, alors «tout deviendrait double » ajoute Socrate. Cela est un point bien important : le contraste entre le modèle et l'image n'est pas le manque de quelques caractères, mais la différence de nature, et non de degrés, entre les deux. Une image, dès lors qu'elle est une image, ne peut pas répliquer

\footnotetext{
${ }^{63}$ Aristote, Métaph., A 6, 987b11-14.

${ }^{64}$ Cfr. Vlastos 1973, p. 351.

${ }^{65}$ Platon, Cratyle, 432a-d, trad. E. Chambry.
} 
exactement son Modèle. «A reflection of a horse is not a horse at all » écrit Petterson ${ }^{66}$. Le statut de l'image $f$ est autre du statut d'un vrai $F$. Patterson insiste sur le fait que c'est précisément parce que l'image de $F$ est différente de $F$ qu'elle peut être image. Autrement dit, le « principe de non identité » est constitutif de l'essence de l'image : « it is one condition on being an image $\mathrm{F}$ that it not be a real $\mathrm{F} »{ }^{67}$. D'une certaine manière, il y a une contrepartie positive dans cette relation : une image de Cratyle n'est pas une image de Simmias. Ainsi, de même que le « principe de non-identité » est constitutif de la nature de l'image, l'identité « d'une certaine manière » est essentielle à la réalité de

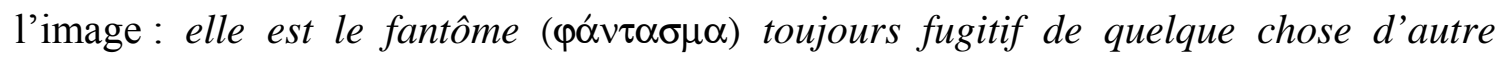

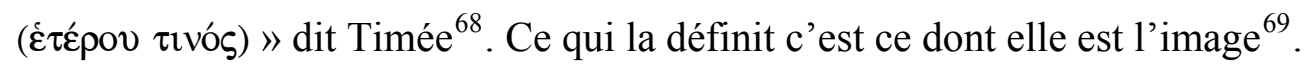

Enfin, il y a dans le Sophiste une définition capitale de l'image qui réconcilie la

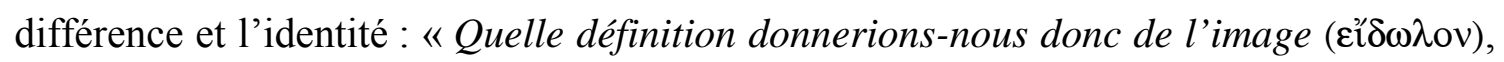
étranger, sinon qu'elle est un autre objet pareil copié sur le vrai ? ${ }^{70}$. Définition à laquelle Théétète ajoute vite une précision : cet « objet pareil» n'est pas un vrai, mais un ressemblant (દ̉otKós) ${ }^{71}$. A la fois identique et différente, nous pourrons définir l'image par sa ressemblance à son modèle, mais il faut préciser une chose importante :

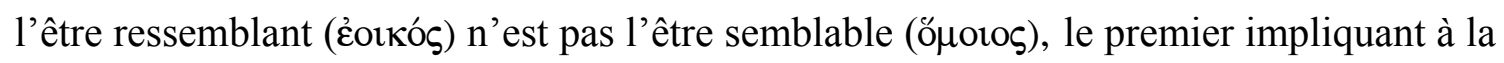
fois identité et différence, le second impliquant similarité.

A la suite de la définition de l'image comme ressemblance, l'étranger du Sophiste se pose la question du statut de cette image, car, si celle-ci n'est pas un « objet vrai », elle n'est pas pour autant irréelle. Théétète admettant que le vrai est réel et que le non-vrai est le contraire du vrai est obligé de reconnaître que l'image, n'étant pas un objet vrai, est «un irréel non-être », qui «a quelque être pourtant», ayant un « réel être de ressemblance ». Ce genre d'être glisse de l'être au non-être ${ }^{72}$. L'image n'est donc pas seulement identique et différente de son modèle, elle est aussi à la fois irréelle et réelle. L'objet ressemblant ( đò ع̇oukós) tire sa réalité d'un «entrelacement de l'être au non-

\footnotetext{
${ }^{66}$ Patterson 1985, p. 3; cfr. p. 20.

${ }^{67}$ Patterson 1985 , p. 39.

${ }^{68}$ Platon, Timée, 52c.

${ }^{69}$ Cfr. Patterson 1985, p. 42.

${ }^{70}$ Platon, Sohpiste, 240a.

${ }^{71}$ Platon, Sophiste, $240 \mathrm{~b}$.

${ }^{72}$ Platon, Sopiste, 240a-c. On retrouve le même embarras à exprimer ce genre dans le livre X de la République, 597a. Si les protagonistes du Sophiste expriment cette ambivalence par la notion de ressemblance, ceux de la République en appellent à l'apparence (X 596e; cfr. 597a-e).
} 
être » dit Théétète. Mais en réalité c'est la réflexion sur le non-être que sera éclairante. Les protagonistes du Sophiste accepteront de poser le non-être, à condition de le comprendre comme l'autre de l'être ${ }^{73}$. L'entrelacement de l'être au non-être est entrelacement du même à l'autre. L'image de Cratyle est à la fois Cratyle «d'une certaine manière » parce que n'est pas l'image de Simmias, et tout autre chose que Cratyle parce qu'elle n'est que l'image ; la même chose est dire que cette image est et n'est pas à la fois : l'image n'est pas puisque n'est pas le modèle, mais elle est autre que le modèle. Ce passage est particulièrement intéressante puisque Platon donne une place au non-être parmi les êtres, et donc donne une réalité à l'image ${ }^{74}$. Brochard lit dans ce texte que «le non-être existe au même degré que l'être ${ }^{75}$. Une telle lecture ne nous semble pas fondée : dire que l'image trouve place parmi les êtres ne signifie pas que l'image a le même degré que son modèle. Pour ce que nous concerne, nous pouvons seulement retenir de ce texte que Platon accepte la réalité de l'image. L'image est certes réelle, mais elle tire sa réalité du modèle dont elle est image, ainsi comme le portrait de Simmias ne peut pas exister si Simmias n'avait jamais existé ${ }^{76}$. L'image est un entredeux, un presque rien qui est quelque chose par renvoi à autre chose qu'elle-même ${ }^{77}$.

Il faut voir maintenant si le statut des participants de l'hypothèse du jeune Socrate est analogue à celui de l'image que nous venons d'élucider.

\section{$\S 5)$ Le statut des participants}

Le seul point sur lequel les commentateurs modernes sont d'accord sur le problème de la participation c'est celui que Vlastos nomme principe de « Non-Identité » (NI) : « If anything has a given character by participating in a Form, it is not identical with that Form $»^{78}$. Ce principe est attesté par Platon lui-même dans la deuxième partie du Parménide: «n'est pas parce qu'elles sont différentes de l'un que, dans le cadre de la participation, les parties participent de l'un ? ${ }^{79}$. Or, le rapport de participation

\footnotetext{
${ }^{73}$ Platon, Sopiste, 256d-257c; 257b-c.

${ }^{74}$ Platon, Sopiste, 258a.

${ }^{75}$ Brochard 1926, p. 145.

${ }^{76}$ Telle est l'idée qu'exprime la définition de l'image du Timée 52c.

${ }^{77}$ Cfr. Cherniss 1956, p. 59 n. 7.

${ }^{78}$ Vlastos 1973, p.351; cfr. Brisson 1994, p. 56; Brunschwig 1985.

${ }^{79}$ Platon, Parm., 158a-b. Ce texte est en accord avec tous les textes marquant l'opposition entre les Forme set les participants: République 476c-d ; Phédon 74c ; Lettre VII 342c-d.
} 
implique aussi quelque chose en commun entre les deux termes. R. A. Patterson souligne ce point : «It would be quite misleading to say simply that sensibles f are not a $\mathrm{f}$ at all [...] it is equally important that sensibles do have some claim to be called $\mathrm{F}$ rather than $G^{\prime}{ }^{80}$. En effet Parménide dans la deuxième partie du dialogue dit «les nonuns ne participent pas davantage à l'un ; sans quoi en effet, ils seraient non pas nonuns, mais uns d'une certaine façon ${ }^{81}$. Si en effet le fait, pour une chose sensible, de participer à une Forme $\mathrm{F}$, donne le caractère $\mathrm{f}$ à cette chose, c'est qu'il y a, d'une certaine manière, une identité. Identité non pas à strictement parler entre la chose f et la Forme F, mais entre le caractère que revêt la chose et celui dont la Forme est caractéristique. Brisson explique que la participation «suppose la possession commune d'une ou plusieurs caractéristiques et donc le rejet de la différence absolue $»^{82}$. Nous sommes d'accord avec Brisson qu'il n'y a pas une «différence absolue », mais la formule de Brisson nous semble difficilement acceptable, puisque il est délicat de parler de caractéristique commune entre une Forme et un sensible. Nous préférons parler en termes de ressemblance. Le Phédon 74c-d évoque la ressemblance en insistant sur la différence entre l'Egalité et les choses égales; en effet, on ne peut pas parler de réminiscence s'il n'y a pas la moindre ressemblance entre les Formes et les sensibles. Littéralement le texte dit que les sensibles sont tantôt semblables (öhotos) tantôt dissemblables $\left(\dot{\alpha} v \operatorname{Lot}_{\mu} \mathrm{\zeta}^{83}\right)^{83}$. En tout cas, le dialogue qui nous permet de saisir au mieux le statut des sensibles par rapport aux modèles est le Timée, où Platon ne se contente pas seulement d'exprimer l'ambiguïté du rapport, mais il en rend compte en faisant intervenir un nouvel élément: le réceptacle $(\chi \omega \dot{\omega} \rho)^{84}$. Le premier «genre d'être » qui participe à l'ordre du monde est la Forme intelligible qui est inengendrée, immuable, incorruptible. Le second genre est la réalité sensible, «qui porte le même nom que la première réalité et qui lui est semblable (óhoเov)» bien qu'elle soit engendrée, muable et corruptible. Nous retrouvons ici le problème de l'ambivalence du statut du sensible, mais dans ce cas-là Platon fait intervenir un troisième genre d'être : la réalité sensible est certes «semblable à la première », mais elle est enracinée dans le réceptacle, sans lequel elle ne serait pas. Ce troisième genre est nécessaire pour distinguer les deux

\footnotetext{
${ }^{80}$ Patterson 1985, p. 41.

${ }^{81}$ Platon, Parm., 147a.

${ }^{82}$ Brisson 1994, p. 56; cfr. Platon, Parm., 131a, 133d.

${ }^{83}$ Cfr. Platon, Euthydème, 300e-301a.

${ }^{84}$ L'étude qui suit se rapporte à la page $52 \mathrm{du}$ Timée.
} 
premiers, puisque sans cet enracinement des sensibles dans le réceptacle la seconde

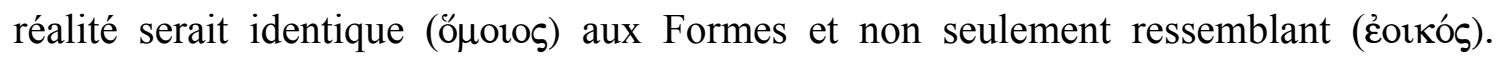
Brisson explique le rôle de la chora de cette façon : « la chose sensible ressemble à la forme intelligible, parce qu'elle en participe. Encore faut-il rendre compte du fait que cette ressemblance n'est pas totale, sinon chose sensible et forme intelligible seraient identiques. D'où l'hypothèse du matériau en quoi se trouve et de quoi se constitue la chose sensible et qui explique sa différence par rapport à la forme intelligible dont elle participe ${ }^{85}$. Ainsi, en faisant intervenir la chora, le Timée permet de justifier la position entre le même et l'autre des participants par rapport aux Formes et il rend compte de leur statut ontologique: de même que l'image, loin d'être véritablement, n'est qu'un "fantôme toujours fugitif ${ }^{86}$, les sensibles qui participent des Formes ne font que passer : «toujours en effet elle (la chora) reçoit toutes choses, et jamais en aucune manière sous aucun rapport elle ne prend une forme qui ressemble à rien de ce qui peut entrer en elle ${ }^{87}$.

Il y a un rapport très étroit entre le fait que les participants sont à la fois dissemblables et semblables à leurs Formes et le fait que celles-ci sont à la fois absentes et présentes «dans» les participants. Dans l'Euthydème Socrate explique que les choses belles sont autre chose que le Beau, bien qu'il se trouve en elles «la présence ( $\pi \alpha \rho \varepsilon \hat{\imath} v \alpha \imath)$ d'une certaine beauté ${ }^{88}$. Comment comprendre une telle présence ? Dionysodore pousse cette idée à l'extrême : «si donc un bøuf se trouve en ta présence, tu es un bœuf, et parce qu'en ce moment je suis en ta présence, tu es Dionysodore ». Dionysodore joue ici sur l'équivocité du terme $\pi \alpha \rho \varepsilon \hat{\imath} v \alpha \iota$ : ce verbe peut signifier être présent au sens d'une proximité physique, ou être en présence de, en sens d'une relation plus abstraite. Or, le deuxième sens est large et donne seulement une idée vague de la relation Forme/sensible, mais le premier sens mène aux apories du Parménide. Même si Parménide n'emploie pas le terme ambigu de Dionysodore et il emploi plutôt $\mu \varepsilon \tau \varepsilon ́ \chi \varepsilon ı v$, $\mu \varepsilon \tau \alpha \lambda \alpha \mu \beta \alpha ́$ veıv, participer signifie dans son discours «avoir une partie de », «être dans ». Cette conception spatiale de la participation mène nécessairement à une aporie ${ }^{89}$. Proclus explique que l'aporie du tout et de la partie ne fait que montrer que l'on ne peut

\footnotetext{
${ }^{85}$ Brisson 1994, p. 251; cfr. Platon, Timée 50d.

${ }^{86}$ Platon, Timée, 52c.

${ }^{87}$ Platon, Timée, 50c.

${ }^{88}$ Platon, Euthydème, 300e-301a.

${ }^{89}$ Brisson 1994, p. 56; cfr. Allen 1983, p. 120.
} 
penser la participation dans un sens concret, à savoir de manière corporelle : « il est impossible que, corporellement, un tout soit en plusieurs sujets [...] Il n'y aurait donc qu'un seul moyen de se rendre compte scientifiquement de la participation : ce serait d'ôter à la participation tout caractère corporel $»^{90}$. En suivant le conseil de Proclus, il faut comprendre la participation dans un sens abstrait, immatériel. L'analogie de l'image peut ici nous aider. Nous avons rencontré le même dilemme dans le Cratyle ${ }^{91}$. Nous avons dit que on ne pouvait pas définir une imitation par des critères quantitatifs. Un portrait de Cratyle n'est pas moins une bonne imitation parce qu'il ne représente que son profil, non plus parce qu'il imite le plus fidèlement possible la plus grande partie de Cratyle. Il s'agit plutôt du fait qu'il émane « une certaine présence » de Cratyle que nous apprécierons le portrait; mais cette présence est toujours accompagné de l'absence. Ainsi, nous pouvons comprendre la participation comme un renvoi à, un signe vers, un rappel : si nous qualifions une chose de belle ou de grande, c'est parce que elle fait signe ( $\sigma \eta \mu \alpha i ́ v \varepsilon \imath)$ vers la Forme de la Beauté ou de la Grandeur.

La pertinence de l'hypothèse formulée par le jeune Socrate semble maintenant évidente. L'analogie de l'image est en effet d'autant plus éclairante que la conception de la participation qu'elle propose s'avère tout à fait en accord avec les textes : dire qu'une chose f participe d'une Forme F, c'est dire qu'elle lui ressemble, à savoir qu'elle est à la fois semblable et dissemblable ; c'est aussi dire qu'elle fait signe vers la Forme F. Avant de terminer, il s'agit d'étudier la validité de la critique de l'hypothèse du jeune Socrate par Parménide.

\section{§ 6) La validité de la critique de Parménide}

Les textes de la République, du Phédon, du Parménide et du Timée ${ }^{92}$ affirment clairement que les Formes sont pensées à part ( $\chi \omega$ pí $)$ des choses sensibles. Or, dire qu'elles sont séparées signifie qu'elles sont distinctes des participants sensibles. Nous qualifions de sens « faible » la séparation comprise comme distinction ${ }^{93}$, par opposition

\footnotetext{
${ }^{90}$ Proclus, Comm. sur le Parm., $\S 73$, vol. 73, vol. II, p. 31 de la trad. Chaignet.

${ }^{91}$ Platon, Cratyle, 432a-d.

${ }^{92}$ Platon, République 476d; Phédon 74; Parménide Ière partie ; Timée 52.

${ }^{93}$ Quand les parties de l'âme sont dites séparées dans la République X, 595b, cela signifie qu'elles sont distinctes les unes des autres.
} 
à un sens «fort» de la même signifiant l'indépendance absolue des Formes ${ }^{94}$. Cette nuance est bien présente en Aristote ${ }^{95}$ et permets de distinguer ce qui est séparé de la matière $^{96}$ de ce qui est subsistant par soi, et qui n'a pas besoin d'autre chose pour exister $^{97}$. Dans la Forme platonicienne le sens faible et le sens fort, séparation de la matière et réalité substantielle coïncident. Malgré cette réunion, la distinction aristotélicienne est importante pour mieux définir le statut des Formes. Il y a séparation au sens fort dans les Formes, mais elle ne peut pas être totale ${ }^{98}$. Sans doute les Formes sont des réalités individuelles qui ont une existence propre; les Formes sont en soi et par soi ( $\alpha$ $\tau \grave{\alpha} \kappa \alpha \theta^{\prime} \alpha$ vi $\left.\alpha\right)$. Loin de se suffire à eux-mêmes, les sensibles sont «pâles reflets $»{ }^{99}$ des modèles intelligibles. Alors que les Formes ne renvoient à rien d'autres

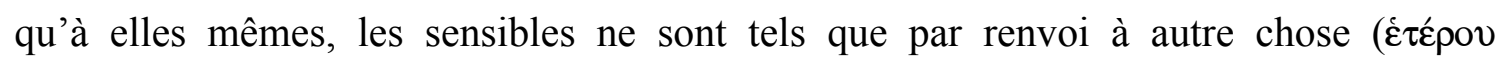
$\tau \imath v o ́ s)^{100}$. Et les sensibles sont doublement dépendants : ils dépendent de leur modèle en amont et du réceptacle dans lequel ils prennent forme en aval ${ }^{101}$. Faces aux Formes en soi et par soi, on ne s'étonnera pas que les sensibles doublement dépendants soient qualifiés d' « inférieurs » par Platon. Dans le Phédon Platon explique que les choses égales ne sont pas au même niveau que 1'Egalité en soi ; elles «aspirent» à devenir semblables à l'Egalité, mais il leur manque beaucoup : elles demeurent « déficientes » $(\dot{\varepsilon} \vee \delta \varepsilon \varepsilon ́ \sigma \tau \varepsilon \rho \alpha)$, «inférieures » $(\varphi \alpha \nu \lambda o ́ \tau \varepsilon \rho \alpha)^{102}$. Cette infériorité du sensible par rapport aux modèles intelligibles entraîne des conséquences importantes concernant le rapport sensibles/Formes : le sens de la relation de participation en découle directement.

En effet, la séparation au sens «faible» que nous avons définie comme distinction implique sans aucun doute une relation symétrique : dire que les Formes sont séparées des sensibles participants au sens «faible» de distinction implique que les sensibles soient aussi séparés des Formes que celles-ci sont séparées des sensibles. Mais si le sens « faible » implique une réciprocité, il en va en revanche autrement pour le sens « fort » exprimant une indépendance absolue. Formes et sensibles n'ayant pas le même statut

\footnotetext{
${ }^{94}$ Quand Platon définit dans le Phédon 64c la mort comme la séparation de l'âme et du corps, c'est le sens « fort » qu'il faut provilégier.

${ }^{95}$ Cfr. Aubenque 1962, p. 36.

${ }^{96}$ Aristote, De Anima II 1, 413a4.

${ }^{97}$ Aristote, Metaph., $\Delta$ 18, 1022a35.

${ }^{98}$ Cfr. Brisson 1994, p. 33.

${ }^{99}$ Platon, Phèdre, $250 \mathrm{~b}$.

${ }^{100}$ Platon, Timée, 52c.

${ }^{101}$ Cfr. Patterson 1985, p. 120.

${ }^{102}$ Platon, Phédon, 75a-b; cfr. Cratyle 432a-d.
} 
ontologique, les unes sont en soi et par soi, les autres ne sont rien par eux-mêmes, ils ne peuvent pas être réciproquement séparés. Comme l'explique très bien Brisson, seules les Formes sont séparées des sensibles : «Les Formes sont séparées des choses sensibles en ce sens qu'elles peuvent exister indépendamment des choses sensibles. En revanche les choses sensibles ne sont pas séparées des Formes, en ce sens qu'elles ne peuvent exister indépendamment des Formes dont elles ne sont que des images $\gg{ }^{103}$.

Si la séparation Formes/sensibles n'est pas réciproque, alors on ne peut penser qu'à une relation de participation unilatérale et non pas symétrique. Il n'y a de participation que dans le sens sensibles $\rightarrow$ Formes, de même que dans la relation image/modèle c'est l'image que vise, qui a pour fin le modèle. Il nous semble éclairant de définir la participation comme une tension infinie des sensibles vers leur Formes, comme un effort d'assimilation: les sensibles tendent à s'assimiler à leurs modèles. Cette idée est très clairement exposée dans les pages du Phédon, qui soulignent la différence irréductible, la tension, l'effort d'assimilation infini des sensibles, n'atteignant jamais le niveau de perfection des Formes. Ils ne peuvent que se rendre les plus semblables possible à leur modèles. Goldschmidt écrit: «ce sont les sensibles qui s'épuisent en des efforts innombrables et impuissants à reproduire l'éclat de la Forme. La Forme est la plénitude essentielle dans laquelle chaque objet-image ne peut découper que quelques traits, qualités usurpées qu'il ne peut retenir longtemps et sur lesquelles il ne peut jamais faire valoir un droit essentiel ${ }^{104}$. Cette lecture est en accord avec des pages cruciales du

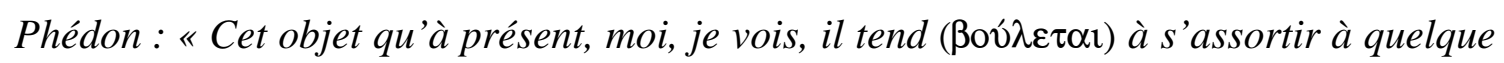
autre réalité ; mais par défaut, il ne réussit pas à être tel qu'est la réalité en question et il lui est, au contraire, inférieur [...]. La vue des égalités nous a donné l'idée que toutes

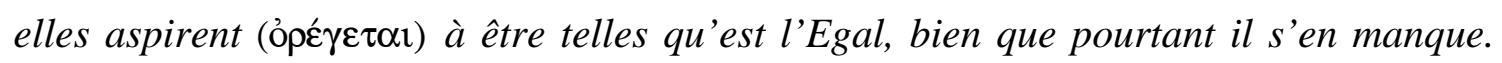
[...] toutes ces égalités ont envie $(\pi \rho \circ \theta v \mu \varepsilon \imath \imath \alpha \alpha)$ de ressembler à l'Egalité même, mais

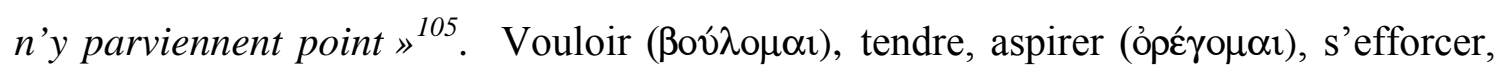
désirer ardemment $(\pi \rho \circ \theta 0 \mu \varepsilon ́ o \mu \alpha \mathrm{l})$, sont des verbes exprimant très vivement le désir ${ }^{106}$. La participation comprise comme effort d'assimilation est en accord avec l'origine du mot « mimêsis ». Dans un article sur l'imitation, G.F. Else explique que le mot $\mu$ í $\mu \eta \sigma r \varsigma$

\footnotetext{
${ }^{103}$ Brisson 1994, p. 42; cfr. Allen 1983, p. 102 et 164.

${ }^{104}$ Goldschmidt 1949, p. 38.

105 Platon, Phédon, 74d-75b.

${ }^{106} \mathrm{Cfr}$. Platon, Théétète, $176 \mathrm{~b}$.
} 
vient du verbe $\mu \mu \varepsilon \varepsilon_{0} \mu \alpha$, celui-ci venant du nom du mime-acteur, le $\mu \hat{\imath} \mu$ os. «the original sphere of mimêsis - or rather of mimos and mimeisthai - was the imitation of animate beings, animal and human, by the body and the voice (...), rather than by artefacts such as statues or sculptures. In other words, these terms originally denoted a dramatic or quasi-dramatic representation, and their extension to non dramatic forms like painting and sculpture must have been a secondary development. (...) this extension had taken place by Aeschylus' time at the latest" ${ }^{\prime 107}$. Bref, le sens premier est actif, se rendre soi-même semblable à un modèle, c'est-à-dire mimer, se faire imitation, tandis que le sens dérivé est passif, être une imitation, être le résultat du travail d'imitation. Comprise comme tension, la participation est donc très proche de l'imitation au sens premier. Dire que les sensibles participent aux Formes, dire qu'ils tendent vers leurs Modèles, qu'ils s'efforcent de se rendre semblable à eux, ou dire qu'ils les imitent, cela signifie pour nous la même chose. La dernière formulation a le mérite d'exprimer le caractère unilatéral de la relation sensibles/Formes, au même temps que la reconnaissance de la dissymétrie de la relation Formes/sensibles met un terme au regressus infini ${ }^{108}$. Comme justement reconnaît Brisson : « la seule façon d'échapper à cette remontée est de reconnaître la différence de statut ontologique des Formes et des choses sensibles. (...) Cela accepté, il faut en outre admettre que la ressemblance des choses sensibles aux Formes est asymétrique ${ }^{109}$. Est donc Parménide même qui engendre le regressus quand il affirme : " une autre Forme surgira encore, et jamais ne cessera l'apparition d'une Forme chaque fois nouvelle, à supposer que cette Forme soit semblable (öhotov) à ce qui en participe $»{ }^{110}$. Le commentaire d'Allen est le suivante : « separation is not a symmetrical relation (...) : Sensibles, as wholly dependant images of Ideas, are neither individual nor substantial, and if this is once granted, the way is open to inquire whether the likeness of sensibles to Ideas is not asymmetrical as separation is asymmetrical. (...) the Likeness regress fails by reason of the failure of the symmetry assumption $\gg{ }^{111}$. En effet, les sensibles et les Formes ne partagent pas le

\footnotetext{
${ }^{107}$ Else 1958, p. 58. Cfr. aussi l'introduction à la Poétique d'Aristote par R. Dupont-Roc et J. Lallot dans laquelle les auteurs, justifiant leur traduction de mimesis par représentation, soulignent le sens originellement théâtral du mot (p. 18).

${ }^{108}$ Platon, Parm., 132c-133a.

109 Brisson 1994, p. 42; cfr. Patterson 1985, p. 55.

110 Platon, Parm., 133a.

111 Allen 1983, p. 164.
} 
même statut ontologique ${ }^{112}$. On ne peut pas dire que la Forme est semblable à une chose sensible sans nier la définition même de la Forme; quel est l'intérêt même de 1'hypothèse des Formes si elles sont les doubles des sensibles ${ }^{113}$, ce qu'implique l'adjectif employé par Parménide, ómorov. Le fait que le jeune Socrate accepte un tel présupposé témoigne d'un manque d'expérience de sa part.

Proclus, considérant que le procédé du dialogue est maïeutique plutôt que réfutatif, comment ainsi l'argument de Parménide : « si le sensible est semblable à l'intelligible, il ne faut pas convertir le rapport, et dire que celui-ci est semblable à celui-là [...]. En effet, si l'on accorde que le participé et le participant sont semblables l'un à l'autre comme le paradigme et celui qui est fait à son image, il y aura avant eux quelque autre chose qui les rendra semblables, et cela à l'infini. Pour répondre à cela, Socrate aurait dû dire qu'il y a deux sortes de semblable, le semblable qui est au même rang d'ordre que son semblable, et le semblable qui est abaissé par rapport à son archétype. [...] Parménide montre qu'il ne faut pas que la relation de ses espèces aux choses d'ici-bas soit convertible ${ }^{114}$. Le glissement de sens de semblable ne se fait pas sens un subtil glissement dans le langage de Parménide, produisant ainsi un effet de symétrie. Comme le fait remarquer Patterson ${ }^{115}$, Parménide substitue aux termes de Socrate exprimant la ressemblance un terme indiquant la similitude. En effet Socrate dit seulement que les

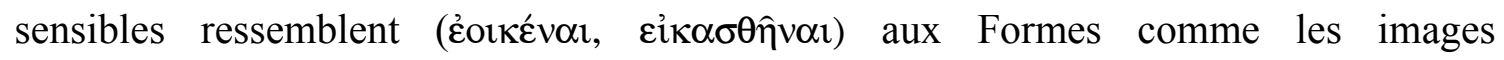

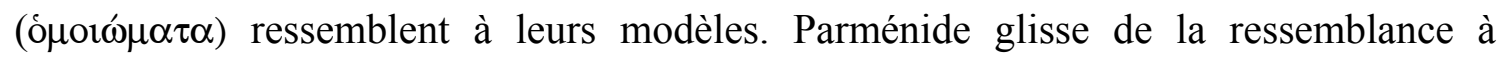
l'identité, en déduisant que deux choses sont semblables du fait qu'elles se ressemblent : «Si quelque chose alors ressemble (ह้orkev) à une Forme, est-il possible que cette

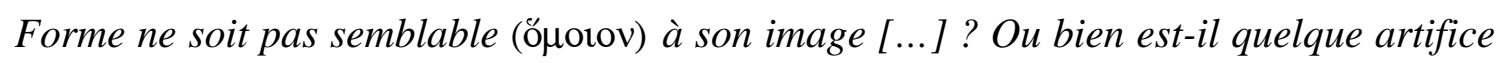

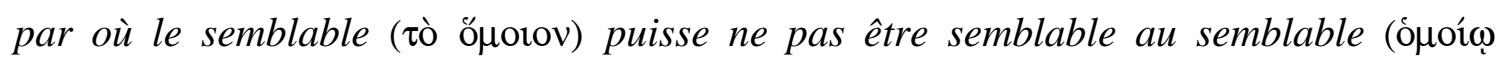

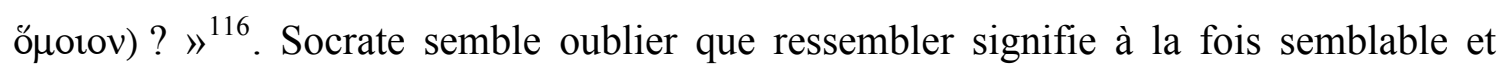
dissemblable et se laisse ici entraîner dans le sophisme de la ressemblance réciproque.

Avant de conclure, il faut dire encore quelque chose à propos de l'Argument du Troisième Homme ${ }^{117}$, car on a vu qu'il menaçait l'hypothèse de Socrate. G. Vlastos, qui

\footnotetext{
${ }^{112}$ Cfr. Goldschmidt 1947, pp. 43-44; Platon, République, V 476c.

${ }^{113}$ Cfr. Patterson 1985, p. 56.

114 Proclus, Comm. sur le Parm., § 79, p. 70-71, vol. II de la trad. Chaignet.

115 Patterson 1985, p. 58.

${ }^{116}$ Platon, Parm., 132d5-8.

${ }^{117}$ Platon, Parm., 132a-b.
} 
a étudié précisément cet argument dans sa forme logique ${ }^{118}$ a mis en évidence deux prémisses qu'il faut supposer pour que l'argument mène effectivement au regressus :

1) la "self-predication" (SP) : « The Form corresponding to a given character itself has that character ${ }^{119}$. L'argument présuppose que la Forme de la Grandeur, par exemple, puisse être elle-même grande.

2) Le principe de Non-Identité (NI).

La prémisse 2) ne pose pas de problèmes, il en va autrement pour la prémisse de l'auto-prédication. En effet Platon a écrit des formules qui peuvent être lues comme des affirmations de auto-prédication. Dans le Protagoras on peut lire que la Justice est juste et la Sainteté est sainte, et dans le Banquet on trouve que la Beauté est belle ${ }^{120}$. En vérité, on ne peut pas parler vraiment de prédication dans ces cas-là. On ne peut pas dire que la Forme du juste est juste comme Socrate est juste. Le point essentiel est que la relation sensibles/Formes est dissymétrique et que donc le regressus de la ressemblance n'est pas valide : le même vaut aussi pour réfuter l'auto-prédication des Formes. Il s'agit de l'irréductible différence de nature entre les Formes qui sont $\alpha$ $\tau \grave{\alpha} \kappa \alpha \theta$ ' $\alpha$ $\tau \grave{\alpha}$ et les

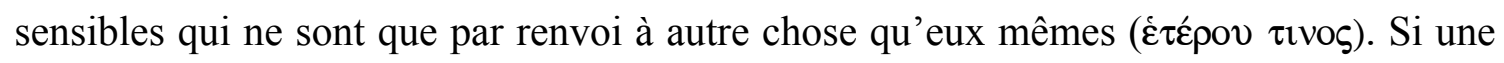
Forme, étant parfaite, est par elle-même tout ce qu'elle peut être, on ne peut pas dire qu'elle est grande de la même manière qu'une chose, celle-ci n'étant telle que par participation à la Forme du Grand, est grande : la Grandeur est Grandeur, mais elle n'est pas grande ${ }^{121}$. Cette idée est présente dans le Banquet, Diotime enseignant la différence entre les choses belles et la Beauté ${ }^{122}$ et dans 1'Hippias Majeur, quand Hippias fait l'erreur de définir la Beauté par l'exemple d'une belle femme ${ }^{123}$.

Cherniss explique de cette façon : «the idea is that which the particular has as an attribute, the «third man » is illegitimate as an argument against the ideas because idea and particular cannot be treated as homogeneous members of a multiplicity $\gg{ }^{124}$. Quand Parménide réunit dans un même ensemble les Formes et les particuliers, en posant une seconde Forme du Grand $\mathrm{G}^{2}$ sous laquelle sont subsumées les choses grandes et la

\footnotetext{
118 Vlastos 1973.

${ }^{119}$ Vlastos 1973, p. 351.

${ }^{120}$ Platon, Protagoras 330c-331b; Banquet 211a-b.

${ }^{121}$ Cfr. Allen 1983, p. 142.

${ }^{122}$ Platon, Banquet, $211 \mathrm{a}$.

${ }^{123}$ Platon, Hippias Majeur, 287d; cfr. République, 476d.

${ }^{124}$ Cherniss 1944, p. 298.
} 
première Forme $\mathrm{G}^{125}$, il fait deux erreurs, offensant la métaphysique platonicienne d'une part et les règles de la logique d'autre part. En effet, réunir les Formes et les sensibles dans le même ensemble implique de nier la définition des Formes $\alpha$ $\tau \grave{\alpha} \kappa \alpha \theta$ ' $\alpha$ $\tau \grave{\alpha}$, et d'ignorer les règles de la logique en plaçant dans un même ensemble des éléments qui n'ont rien en commun ${ }^{126}$. Puisque la prémisse nécessaire à la validité de l'Argument du Troisième Homme est l'auto-prédication et elle n'est pas soutenable dans le système platonicien, on ne peut pas dire que l'argument de Parménide est valide. Prédiquer c'est attribuer une qualité à un sujet afin de le définir, ce qui implique que le sujet ne se définisse pas par lui-même. Mais on ne peut pas parler de prédication à propos des Formes, qui sont, pas définition, par elles-mêmes ce qu'elles sont. Quand Diotime affirme que la Beauté est belle, il faut comprendre que la Beauté n'est que ce qu'elle est : la Beauté est Beauté. L'auto prédication des Formes est une tautologie ${ }^{127}$.

\section{$\S 7)$ Conclusion}

L'hypothèse du jeune Socrate du Parménide présentant les Formes comme des Modèles dont les images sont les sensibles, implique que le rapport de participation se comprends comme un rapport de ressemblance et d'imitation. Cet hypothèse s'est avérée particulièrement pertinente. En effet le rapport image/Modèle, tel qu'il est conçu par Platon, nous a permis d'éclairer le rapport participant/Forme, dont aucune définition n'est clairement établie. Dans cette analogie il y a deux points essentiels: 1) la dissymétrie du rapport sensible/Forme, à savoir la différence de nature entre les deux éléments, qui comporte l'unilatéralité de la relation : seuls les sensibles participent des Formes ; 2) la conception de la participation comme imitation au sens actif, ce que l'on retrouve dans le verbe mimer : les sensibles imitent, à savoir s'efforcent de ressembler aux Formes intelligibles parfaits.

Ces deux points nous permettent de mettre en évidence l'inconsistance des deux arguments de Parménide menant à un regressus : le dilemme de la participation et l'Argument du Troisième Homme. La participation comprise comme imitation nous prévient contre le risque d'interpréter d'une façon concrète le rapport sensible/Forme.

\footnotetext{
${ }^{125}$ Platon, Parm., 132b.

${ }^{126}$ Cfr. Goblot 1929, pp. 478-479.

${ }^{127}$ Cfr. Cherniss 1965, pp. 370-371.
} 
Journal of Ancient Philosophy Vol. II 2008 Issue 2

Quant au regressus de la ressemblance présupposant la réciprocité du rapport de ressemblance, et à celui de la Grandeur présupposant que les Formes partagent avec les sensibles le caractère dont elles sont la caractéristique, tous les deux apparaissent comme étant inconsistant en vertu de l'irréductible différence de nature entre les Formes et les sensibles. Si l'on comprend la participation comme un effort d'assimilation, on ne peut pas parler de séparation totale entre le sensible et l'intelligible. Les Formes comme Modèles sont «séparées », transcendantes, mais les sensibles dépendent des Formes dont ils tirent leurs caractères.

Enfin, il n'y a pas chez Platon deux « mondes », l'Intelligible et le sensible. Il n’y a qu'un monde hiérarchisé comprenant différents degrés de réalité, dont l'unité se manifeste sous la forme d'une tension infinie. Le dialecticien est la preuve de l'existence de cette tension. Partir des beautés sensibles pour élever graduellement l'âme jusqu'au Beau en soi, n'est peut-être pas la voie qui propose Diotime dans le Banquet ${ }^{128}$

\section{Bibliographie}

Textes anciens

PLATON. Ouvres complètes, Coll. des Universités de France (G. Budé), Paris, Les Belles Lettres. Nous avons également utilisé les nouvelles traductions avec introduction et notes de L. Brisson pour le Timée et le Parménide, Paris, Flammarion, 1992 et 1994 ; et la traduction de P. Pachet pour la République, Paris, Gallimard, 1993.

ARISTOTE. Métaphysique, traduction et notes de J. Tricot, Paris, Vrin, 1953.

ARISTOTE. Poétique, traduction et commentaire de R. Dupont-Roc et J. Lallot, Paris, Le Seuil, 1980.

PROCLUS. Commentaire sur la République, traduction et notes de A. J. Festugière, Paris 1970.

Id., Commentaire sur le Timée, traduction et notes de A. J. Festugière, Paris 1970.

Id., Commentaire sur le Parménide, traduction et notes de E. Chaignet, Paris 1900.

\section{Etudes modernes}

ALLEN R.E. Participation and predication in Plato's Middle Dialogues, in R.E. Allen (ed.), Studies in Plato's Metaphysics, London 1965.

\footnotetext{
${ }^{128}$ Platon, Banquet 211c ; cfr. Phédon, 74c-d; Phèdre, 250a-b ; 250b ; République VII 502c
} 
Journal of Ancient Philosophy Vol. II 2008 Issue 2

ALLEN R.E. Plato's Parmenides, trad. et analyse, Oxford 1983.

ANNAS J. Introduction à la République de Platon, Oxford 1981.

AUBENQUE P. Le problème de l'être chez Aristote, Paris 1962.

BABUT D. Sur la notion d'imitation dans les doctrines esthétiques de la Grèce Classique, Revue des Etudes Grecques 98 (1985), pp. 72-92.

BRANDWOOD L. A word index to Plato, Leeds 1976.

BRISSON L. Participation et prédication chez Platon, Revue Philosophique de la France et de l'Etranger 4 (1991), pp. 557-569..

BRISSON L., Le même et l'autre dans la structure ontologique du Timée, Paris 1974.

BROCHARD V. La théorie platonicienne de la participation d'après le Parménide et le Sophiste, Etudes de Philosophie Ancienne et de Philosophie Moderne, Paris 1926.

BRUNSCHWIG J. Le problème de la «self-participation» chez Platon, L'art des confins, in Mélanges offerts à M. De Gandillac, Paris 1985, pp. 121-135.

CHERNISS H.F. Aristotle's criticism of Plato and the Academy, Baltimore 1944.

CHERNISS H.F. Timaeus 52c2-5, in Mélanges de Philosophie Grecque offerts à A. Diès, Paris 1956, pp. 49-60.

CHERNISS H.F. The relation of the Timaeus to Plato's later dialogues, in R.E. Allen (ed.), Studies in Plato’s Metaphysics, London 1965.

CONFORD F.M. Plato and Parménides, London 1939.

ELSE F.F., Imitation in the Fifth Century, Classical Philology 53 (1958), pp. 73-90.

FRONTEROTTA F. Methexis. La teoria platonica delle idée e la partecipazione delle cose empiriche. Dai dialoghi giovanili al Parmenide, Pisa 2000.

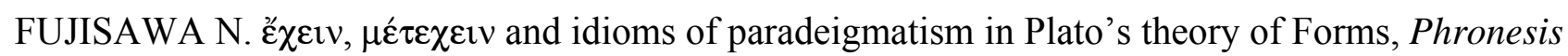
19 (1974), pp. 30-58.

GALLOP D. Image and reality in Plato's Republic, Archiv für Geschichte der Philosophie, 47 (1965), pp. 113-131.

GOBLOT F. L'argument du troisième homme, Revue d'Histoire de la Philosophie 7 (1929), pp. 473-481.

GOLDSCHMIDT V. Le paradigme dans la dialectique platonicienne, Paris 1947.

GOLDSCHMIDT V. La religion de Platon, Paris 1949.

LASSEGUE M. L'imitation dans le Sophiste de Platon, in Etudes sur le Sophiste, recueil publié sous la direction de P. Aubenque, Napoli 1991, pp. 249-265. 
Journal of Ancient Philosophy Vol. II 2008 Issue 2

LEE E.N. On the metaphysics of the image in Plato's Timaeus, The Monist 50 (1966), pp. 341-368. MIGNUCCI M. Plato's third man arguments in the Parmenides, Archiv für Geschichte der Philosophie 72 (1990), pp. 143-181.

NATALI C. La forma platonica è una causa formale?, in G. Damschen, R. Ebskat, A.G. Vigo (a cura di), Plato und Aristoteles sub ratione veritatis. Festschrift für Wolfgang Wieland zum 70. Geburtstag, Göttingen 2003, pp. 158-173.

NEHRLICH G.C. Regress arguments in Plato, Mind 69 (1960), pp. 88-90.

PATTERSON R.A. The unique worlds of the Timaeus, Phoenix 35 (1981), pp. 105-119.

PATTERSON R.A. Image and Reality in Plato's Metaphysics, Indianapolis 1985.

PRIOR W.J. Parmenides 132c-133a and the development of Plato's thought, Phronesis 24 (1979), pp. $230-240$.

SEDLEY D. Platonic causes, Phronesis, 43/2 (1998), pp. 114-132.

TAYLOR A.E. The Parmenides of Plato, Oxford 1934.

VILLELA-PETIT M. La question de l'image artistique dans le Sophiste, in Etudes sur le Sophiste, recueil publié sous la direction de P. Aubenque, Napoli 1991, pp. 55-90.

VLASTOS G. Platonic Studies, Princeton 1973.

VLASTOS G. Reasons and causes in the Phaedo, (1969), maintenant dans Platonic Studies, Princeton 1981.

WAHL J. Etude sur le Parmenide de Platon, Paris 1926.

ZELLER E. Die Philosophie der Griechen in ihrer geschichtlichen Entwicklung, 2.1, Berlin 1922. 